\title{
The Friedreich ataxia GAA repeat expansion mutation induces comparable epigenetic changes in human and transgenic mouse brain and heart tissues
}

\begin{tabular}{|c|c|}
\hline Journal: & Human Molecular Genetics \\
\hline Manuscript ID: & draft \\
\hline Manuscript Type: & 2 General Article - UK Office \\
\hline $\begin{array}{l}\text { Date Submitted by the } \\
\text { Author: }\end{array}$ & $\mathrm{n} / \mathrm{a}$ \\
\hline Complete List of Authors: & $\begin{array}{l}\text { Al-Mahdawi, Sahar; Brunel University, Biosciences } \\
\text { Mouro Pinto, Ricardo; Brunel University, Biosciences } \\
\text { Ismail, Ozama; Brunel University, Biosciences } \\
\text { Varshney, Dhaval; Brunel University, Biosciences } \\
\text { Lymperi, Stefania; Brunel University, Biosciences } \\
\text { Sandi, Chiranjeevi; Brunel University, Biosciences } \\
\text { Trabzuni, Daniah; Brunel University, Biosciences } \\
\text { Pook, Mark; Brunel University, Biosciences }\end{array}$ \\
\hline Key Words: & $\begin{array}{l}\text { FRDA, Friedreich ataxia, Frataxin, GAA trinucleotide repeat, } \\
\text { epigenetics }\end{array}$ \\
\hline
\end{tabular}

\section{๑) ScholarONE" \\ Manuscript Central}


The Friedreich ataxia GAA repeat expansion mutation induces comparable epigenetic changes in human and transgenic mouse brain and heart tissues

Sahar Al-Mahdawi, Ricardo Mouro Pinto, Ozama Ismail, Dhaval Varshney, Stefania Lymperi, Chiranjeevi Sandi, Daniah Trabzuni and Mark Pook*.

Hereditary Ataxia Group, Centre for Cell \& Chromosome Biology and Brunel Institute of Cancer Genetics \& Pharmacogenomics, Division of Biosciences, School of Health Sciences \& Social Care, Brunel University, Uxbridge UB8 3PH UK

Tel: 441895 267243; Fax: 441895 274348; E-mail: Mark.Pook@brunel.ac.uk

*To whom correspondence should be addressed 


\section{ABSTRACT}

Friedreich ataxia (FRDA) is caused by a homozygous GAA repeat expansion mutation within intron 1 of the FXN gene, leading to reduced expression of frataxin protein. Evidence suggests that the mutation may induce epigenetic changes and heterochromatin formation, thereby impeding gene transcription. In particular, studies using FRDA patient blood and lymphoblastoid cell lines have detected increased DNA methylation of specific CpG sites upstream of the GAA repeat and histone modifications in regions flanking the GAA repeat. In this report we show that such epigenetic changes are also present in FRDA patient brain and heart tissues, the primary affected systems of the disorder. Bisulfite sequence analysis of the $F X N$ flanking GAA regions reveals a shift in the FRDA DNA methylation profile, with upstream CpG sites becoming consistently hypermethylated and downstream $\mathrm{CpG}$ sites becoming consistently hypomethylated. We also identify differential DNA methylation at three specific $\mathrm{CpG}$ sites within the $F X N$ promoter and one $\mathrm{CpG}$ site within exon 1. Furthermore, we show by chromatin immunoprecipitation (ChIP) analysis that there is overall decreased histone $\mathrm{H} 3$ and $\mathrm{H} 4$ acetylation together with increased $\mathrm{H} 3 \mathrm{~K} 9$ methylation of FRDA brain tissue. Further studies of brain and heart tissues from our GAA repeat expansion-containing FRDA YAC transgenic mice reveal comparable epigenetic changes to those detected in FRDA patient tissue. We have thus developed a mouse model that will be a valuable resource for future therapeutic studies targeting epigenetic modifications of the FXN gene to increase frataxin expression. 


\section{INTRODUCTION}

FRDA is an autosomal recessive neurodegenerative disorder that is predominantly caused by a homozygous GAA repeat expansion mutation within intron 1 of the $F X N$ gene (1). Normal individuals have 5 to 30 GAA repeat sequences, whereas affected individuals have from approximately 70 to more than 1,000 GAA triplets (2). The GAA repeat shows somatic instability, with progressive expansion throughout life, particularly in the cerebellum and dorsal root ganglia (DRG) (3-5). The effect of the GAA expansion mutation is to reduce the expression of frataxin (6), a mitochondrial protein that acts as an iron chaperone in iron-sulphur cluster and heme biosynthesis (7-9). Frataxin insufficiency leads to oxidative stress, mitochondrial iron accumulation and resultant cell death, with the primary site of pathology being in the large sensory neurons of the DRG and the dentate nucleus of the cerebellum (10). The outcome is progressive spinocerebellar neurodegeneration, causing symptoms of ataxia, dysarthria, muscle weakness, and sensory loss, together with cardiomyopathy, and diabetes. At present there is no effective treatment for FRDA, and affected individuals generally die in early adulthood from the associated heart disease.

Preclinical and clinical trials using antioxidants and iron chelators have demonstrated some limited success in alleviating FRDA heart pathology (11-16). However, a more effective overall therapeutic strategy may be to target the immediate effects of the GAA repeat expansion mutation to restore normal levels of frataxin expression. The exact mechanism by which the GAA repeat 
expansion leads to decreased frataxin expression is unknown, but several models have been put forward. Firstly, it has been suggested that the GAA repeat expansion may adopt abnormal DNA or DNA/RNA hybrid structures that interfere with $F X N$ gene transcription (17-20). Secondly, there is evidence that GAA repeat expansions produce a heterochromatin-mediated gene silencing effect (21). Epigenetic mechanisms, such as DNA methylation and the associated deacetylation and methylation of histones are known to affect gene expression by chromatin remodelling (22), and these epigenetic changes are likely to underpin any GAA repeat-induced heterochromatin-mediated gene silencing effects. In support of this hypothesis, research has recently shown increased DNA methylation of three specific $\mathrm{CpG}$ sites immediately upstream of the expanded GAA repeat sequence in FRDA patient lymphoblastoid cell lines and primary lymphocytes, and one of the three CpG sites was identified as an important enhancer of frataxin expression (23). Other studies have identified specific histone modifications that are associated with gene silencing within the GAA repeat expansion-flanking regions of the FXN intron 1 sequence in FRDA lymphoblastoid cell lines and primary lymphocytes $(23,24)$. These changes include deacetylation of histone $\mathrm{H} 3$ and $\mathrm{H} 4$ lysine residues and increased di- and trimethylation of $\mathrm{H} 3 \mathrm{~K} 9$. Based on the hypothesis that the acetylation state of the core histones is responsible for gene silencing, novel histone deacetylase (HDAC) inhibitor compounds have been developed and have been shown to increase $F X N$ transcription in FRDA lymphoblastoid cells and primary lymphocytes (24). 
These previous epigenetic studies have provided valuable insights into the possible mechanism of GAA-induced transcription inhibition, but they do not address the issue of whether such epigenetic changes are actually present in the most clinically relevant FRDA tissues. Therefore, we decided to investigate epigenetic profiles of the FXN gene in FRDA patient autopsy brain and heart tissue. By bisulfite sequencing and ChIP analysis we now report changes in DNA methylation and histone modifications that are consistent with inhibition of $F X N$ transcription. With a view to future epigenetic-based FRDA therapies, we also investigated the $F X N$ epigenetic profiles within brain and heart tissue from our Y47, YG8 and YG22 FRDA YAC transgenic mouse models (25-27). We find that the GAA repeat expansion-containing FRDA mouse models (YG8 and YG22) exhibit comparable epigenetic changes to those detected in FRDA patient tissue. Therefore, these are excellent FRDA mouse models in which to investigate the therapeutic effects of epigenetically-acting compounds, such as novel HDAC inhibitors or DNA methylation inhibitors.

\section{RESULTS}

FXN gene DNA methylation profiles are distinctly altered in human FRDA brain and heart tissues

A previous investigation of the FXN gene in FRDA patient lymphoblastoid cell lines and blood samples has detected hypermethylation at three specific $\mathrm{CpG}$ sites immediately upstream of the expanded GAA repeat sequence. One of the three CpG sites was further identified as an important enhancer element for 
frataxin expression (23). This same study also reported a lack of any DNA methylation in the promoter region of either FRDA or unaffected cells. However, cultured cells are known to often develop non-physiological DNA methylation profiles. Furthermore, FRDA is a systemic disorder that is known to have differentially affected tissues and cell types. Therefore, we chose to investigate the DNA methylation status in two of the primary affected tissues in FRDA, namely brain and heart. We obtained brain and heart autopsy tissues from an FRDA patient (GAA repeat sizes of 750 and 650 ) and an unaffected individual, and we firstly determined the $F X N$ transcription levels of the samples by quantitative RT-PCR. The FRDA brain and heart samples showed $12 \%$ and $67 \%$ FXN expression, respectively, compared with the unaffected samples (Fig. 1). We then analysed the DNA methylation status of the samples by performing bisulfite sequence analysis of three regions of the $F X N$ gene: (i) a $475 \mathrm{bp}$ sequence that encompasses part of the $F X N$ promoter, exon 1 and start of intron 1, containing $59 \mathrm{CpG}$ sites; (ii) a 286bp sequence upstream of the GAA repeat, containing $8 \mathrm{CpG}$ sites, and (iii) a 275bp sequence downstream of the GAA repeat, containing $12 \mathrm{CpG}$ sites (Fig. 2). A comparison of the bisulfite sequences from the FRDA patient and control brain and heart tissues reveals a certain degree of DNA methylation in all 8 of the upstream GAA CpG sites (Fig. 3C and D) and all 12 of the downstream GAA CpG sites (Fig. 3E and F). However, the data show a consistent shift in the DNA methylation pattern around the GAA repeat in both tissue types. The FRDA upstream GAA CpG sites are comparatively hypermethylated, whereas the FRDA downstream GAA CpG sites 
are comparatively hypomethylated (Fig. 3C-F). The greatest increase in DNA methylation within the upstream GAA region is seen at $\mathrm{CpG}$ site 4 . Significantly increased DNA methylation is also seen at CpG sites 5 and 6 , the latter of which corresponds to the previously described E-box enhancer element (23). We observed 100\% methylation at CpG site 6 in FRDA brain tissue (FXN mRNA level of $12 \%$, Fig.1) compared with $80 \%$ methylation in heart tissue (FXN mRNA level of $67 \%$, Fig.1). Thus, the upstream GAA DNA methylation changes in both FRDA brain and heart are consistent with their proposed roles in inhibition of FXN transcription. However, the finding of decreased DNA methylation in the downstream GAA region (Fig. $3 \mathrm{E}$ and $\mathrm{F}$ ) is somewhat unexpected, since all of the $12 \mathrm{CpG}$ sites fall within an Alu repeat sequence and such sequences are usually repressed by heavy DNA methylation.

Another particularly interesting finding was the identification of differential DNA methylation at three specific CpG sites within the FXN promoter (sites 5, 7 and 8) and one CpG site within exon 1 (site 23) (Fig. 3A and B). All of the other $55 \mathrm{CpG}$ sites in the total of $59 \mathrm{CpG}$ sites analysed show complete lack of DNA methylation, as to be expected for a CpG island that is situated at the start of a gene. CpG sites 5, 7 and 8 show incomplete methylation in the unaffected heart, but complete methylation in the FRDA heart (Fig. 3B). Therefore, these CpG sites may be involved in reducing initiation of $F X N$ gene transcription in FRDA heart. However, the DNA methylation pattern is different in brain tissue. Here we identified approximately 20\% DNA methylation at all of the four CpG sites in the unaffected tissue, but very little change, and even a decrease, in DNA 
methylation in FRDA tissue (Fig. 3A). Furthermore, the fact that we have identified some degree of DNA methylation at all in this region contrasts with the previous report that DNA methylation is absent in the FXN promoter region of both FRDA and unaffected lymphoblastoid cells (23). Therefore, we have shown that the influence of DNA methylation on $F X N$ gene expression is likely to be complex, with some similarities (CpG site usage) but also some distinct differences (degree of $\mathrm{CpG}$ methylation) identified between different somatic tissues.

\section{FXN gene histone modifications are altered in human FRDA brain tissue}

Previous studies of the promoter, upstream GAA and downstream GAA regions of the $F X N$ gene have identified specific histone modifications that are associated with gene silencing within the GAA repeat expansion-flanking regions of the $F X N$ intron 1 sequence in FRDA lymphoblastoid cell lines and primary lymphocytes $(23,24)$. We have now investigated acetylated histone $\mathrm{H} 3$ and $\mathrm{H} 4$ and methylated histone $\mathrm{H} 3 \mathrm{~K} 9$ modifications by ChIP analysis of the $F X N$ promoter, upstream GAA and downstream GAA regions (Fig. 2) in autopsy brain tissues from an FRDA patient and an unaffected individual. Our results show overall decreased histone $\mathrm{H} 3$ and $\mathrm{H} 4$ acetylation of FRDA brain tissue, particularly in the downstream GAA region (Fig.4). All of the 6 acetylated histone residues that we have examined show a GAA-induced gradient of comparative acetylation that is highest in the FXN promoter and lowest in the downstream GAA region. The single most altered histone residue is $\mathrm{H} 3 \mathrm{~K}$, which exhibits progressive 
decreases in acetylation to comparative levels of $63 \%, 35 \%$ and $9 \%$ in the $F X N$ promoter, upstream GAA and downstream GAA regions, respectively. There is also a consistently increased H3K9 di- and tri-methylation of FRDA brain tissue in all three of the $F X N$ gene regions (Fig. 4). These changes concur with the previous findings of increased $\mathrm{H} 3 \mathrm{~K} 9$ di- and tri-methylation in the upstream GAA region of other cell types $(23,24)$. However, we have now extended these studies to show that in FRDA brain the H3K9 di- and tri-methylation spreads to both $F X N$ promoter and downstream GAA regions.

\section{DNA methylation profiles of $F X N$ transgenic mouse brain and heart tissues resemble the profiles of human tissue}

Having determined the epigenetic profiles around the human $F X N$ gene, we then investigated the epigenetic profiles of the $F X N$ transgene in brain and heart tissue isolated from YG8 and YG22 GAA repeat expansion-containing FXN YAC transgenic mice (26) compared with Y47 normal-sized GAA repeat-containing FXN YAC transgenic mice (27). Initial determination of FXN transgene expression showed YG8 (90+190 GAA repeats) and YG22 (190 GAA repeats) to have decreased mRNA levels of approximately $26 \%$ and $35 \%$ in brain and $57 \%$ and $56 \%$ in heart compared with Y47 (Fig. 5). Thus, inhibition of FXN expression in transgenic mouse brain was not as severe as the $12 \%$ observed in the 750/650 GAA repeat-containing human FRDA brain (Fig. 1), whereas levels of FXN expression in heart were more comparable. DNA methylation analysis was then performed on the single GAA repeat expansion-containing YG22 GAA 
repeat transgenic mouse compared to the $\mathrm{Y} 47$ non-GAA repeat control. As the mouse transgenes consist of entire human $F X N$ gene sequence, we were able to investigate the DNA methylation profiles of exactly the same three regions of the FXN gene that we had previously analysed in human tissue (Fig. 2). Our data show that the DNA methylation profiles of upstream GAA regions of both YG22 transgenic mouse brain and heart tissues closely resemble those found in human tissues (Fig. 6C and D). Namely, there is a consistent hypermethylation of the upstream GAA region induced by the GAA repeat expansion, with the most prominent hypermethylation at CpG sites 4, 5 and 6. However, the degree of DNA methylation at CpG sites 4 and 6 in YG22 transgenic mouse brain tissue is less than that observed in FRDA human brain tissue, which is consistent with the comparative FXN expression levels of $35 \%$ and 12\%, respectively (Figs. 1 and 5). The downstream GAA region differs from the human situation in that there is hypermethylation at all CpG sites, which is retained upon introduction of the GAA repeat expansion (Fig. 6E and F). Thus, there is no GAA-induced decrease in DNA methylation as detected in the human tissues. The promoter/exon 1 regions of the FXN transgenes in both mouse brain and heart tissues show a similarity to the human tissues in that DNA methylation is found at only four specific CpG sites: 5, 7, 8 and 23 (Fig. 6A and B). However, the changes in the DNA profiles of these four CpG sites upon introduction of the GAA repeat expansion differ markedly from those found in the human tissues. This time, the brain tissue shows an overall increase in DNA methylation, whereas the heart tissue shows an overall decrease in DNA methylation. Assessment of the entire mouse DNA 
methylation data indicates a similar overall DNA methylation profile around the start of the FXN gene that is consistent with inhibition of $F X N$ transcription. However, there are also some specific differences, which may result from epigenetic-control or transcriptional-control variations between the human and the mouse that will require further investigation.

\section{Histone modifications of $F X N$ transgenic mouse brain tissue are comparable to histone modifications of human tissue}

Acetylated histone $\mathrm{H} 3$ and $\mathrm{H} 4$ and di- and tri-methylated histone $\mathrm{H} 3 \mathrm{~K} 9$ modifications were detected by ChIP analysis of the three regions of the FXN transgene (Fig. 2) in brain tissue isolated from both YG8 and YG22 GAA repeat expansion-containing FXN YAC transgenic mice (26) and Y47 normal-sized GAA repeat-containing FXN YAC transgenic mice (27). Our results show overall GAA repeat-induced decreases in histone $\mathrm{H} 3$ and $\mathrm{H} 4$ acetylation and increases in H3K9 methylation for both YG8 and YG22 transgenic mice (Fig. 7), as we previously identified in human FRDA tissue (Fig. 4). However, the level of deacetylation in the transgenic mouse tissue was not as great as that seen in the human tissue, possibly as a consequence of the smaller transgenic GAA repeat expansion sizes (190+90 for YG8 and 190 for YG22, compared with 750/650 for FRDA patient). Also, $\mathrm{H} 4 \mathrm{~K} 16$ acetylation is actually increased in all three FXN transgene regions of both $Y G 8$ and $Y G 22$ compared with $Y 47$, which is different to the finding in human tissues. The greatest consistent histone residue changes that we found between the non-GAA (Y47) and both of the GAA (YG8 and YG22) 
transgenic brain tissue samples were decreases in acetylated H3K9 and increases in di- and tri-methylated $\mathrm{H} 3 \mathrm{~K} 9$. The $\mathrm{H} 4 \mathrm{~K} 12$ residue also showed a significant degree of deacetylation, but only in the YG8 transgenic tissue. All of these major histone residue changes in mouse brain tissue reflect the GAA repeat-induced histone residue changes that we detected in human tissue. Furthermore, as with the human samples, we similarly identified a GAA repeatinduced gradient of decreased H3K9 acetylation in both YG8 and YG22 transgenic mouse tissues, with the highest comparative levels of acetylation in the $F X N$ promoter and the lowest comparative levels in the downstream GAA region. The increases in $\mathrm{H} 3 \mathrm{~K} 9$ di- and tri-methylation were consistent throughout all of the three $F X N$ gene regions in both $Y G 8$ and $Y G 22$ transgenic mice, once again agreeing with our findings in human FRDA tissue.

\section{DISCUSSION}

For the consideration of future FRDA therapy, it is first essential to understand the mechanism of GAA-induced inhibition of $F X N$ gene transcription. Previous studies of FRDA have implicated epigenetic changes, including the detection of increased DNA methylation of specific $\mathrm{CpG}$ sites upstream of the GAA repeat and histone modifications in regions flanking the GAA repeat that are both consistent with transcription inhibition $(23,24)$. However, no DNA or histone methylation changes have previously been identified in the FXN promoter or downstream GAA regions, and clinically relevant FRDA brain and heart tissues have not previously been investigated. Different trinucleotide repeat expansion 
mutations have been shown to induce cis-acting epigenetic changes in several other human disorders $(28,29)$. Thus, DNA methylation of the CGG repeat upstream of the FMR1 gene has been identified as a main epigenetic switch in Fragile X syndrome, with histone acetylation playing an ancillary role (30). Decreased Sp1 interaction associated with DNA hypermethylation upstream of the CTG repeat in the DMPK gene has also been reported for congenital myotonic dystrophy type 1 (29). Furthermore, both CTG and GAA repeat expansions have been shown to induce similar heterochromatin formation by position effect variegation studies of transgenic mice (21). However, it is still uncertain if different trinucleotide repeat sequences produce similar overall epigenetic effects or not.

Our investigations of the $F X N$ gene in both FRDA human and transgenic mouse brain and heart tissues have now confirmed the presence of previously described DNA methylation changes (23) in the upstream GAA region of these clinically important tissues. Furthermore, our data have revealed an overall shift in the DNA methylation profile, moving from hypomethylation in the downstream GAA region towards hypermethylation in the upstream GAA region. This shift in DNA methylation profile could be explained by the known position of the GAA repeat within an Alu sequence, since Alu sequences have been shown to act as methylation centres leading to bi-directional spread of DNA methylation (31). Thus, the hypermethylation detected in the FRDA upstream GAA region may be due to the GAA repeat mutation enhancing the effect of a putative methylation centre at the 5' end of the Alu sequence. At the same time, the addition of the 
GAA repeat sequence would put extra distance between the methylation centre at the 5' end of the Alu sequence and the downstream GAA region. This may impede the spread of methylation to the downstream region when the distance is large enough ( $2.25 \mathrm{~kb}$ for 750 human GAA repeats), but not when the distance is smaller (600bp for 190 transgenic mouse GAA repeats).

We have additionally identified differential DNA methylation at four specific CpG sites within the $F X N$ promoter and exon 1 regions that have not previously been reported. The three CpG sites within the promoter region (sites 5, 7 and 8) are immediately upstream of the ATG translation start site, at nucleotide positions $-27,-18$ and -11 , respectively. CpG sites 5 and 7 are also contained within Sp1 transcription factor binding sites (32). Interestingly, the region between -64 and the start of translation has previously been suggested to contain sequences important for positive regulation of frataxin production, although no candidate sequences were identified (33). Therefore, the 3 differentially methylated $\mathrm{CpG}$ sites that we have now uncovered in the $F X N$ promoter, and in particular the two Sp1 recognition sites, are likely to represent these important regulatory sequences.

By comparison with other instances of trinucleotide repeat-induced DNA methylation changes that inhibit transcription $(28,29)$, one would have predicted general hypermethylation to be associated with the FRDA GAA repeat expansion mutation. However, we actually identified three occurrences in human tissues (promoter and downstream GAA regions in brain, and downstream GAA region in heart) and one occurrence in mouse tissues (promoter region in heart) where 
there was in fact GAA repeat expansion-induced decrease in DNA methylation. This suggests the possible occurrence of demethylation and resultant active $F X N$ gene expression, at least for some cells within the tissue. DNA demethylation has previously been shown to occur both passively due to DNA replication upon cell division (34) and actively in a process that may involve RNA (35), although the DNA demethylating activity has yet to be identified. DNA demethylation has also previously been associated with processes of DNA damage and repair. The formation of 8-OH-dG by oxidative DNA damage has been shown to affect the activity of human DNA methyltransferase and inhibit CpG methylation (36), and DNA demethylation has also been shown to occur as a result of homologous recombination repair of DNA damaged by double-strand breaks (37). However, DNA demethylation has not previously been considered for FRDA. A close inspection of our data reveals potential DNA demethylation in the FXN promoter region only when $\mathrm{CpG}$ site 6 of the upstream GAA region is $100 \%$ methylated. Therefore, we now propose that the shutdown of transcription due to major epigenetic changes at the upstream GAA region may result in attempts to upregulate $F X N$ transcription by Sp1 binding and subsequent DNA demethylation in the promoter region. In support of this proposal, Sp1 binding is known to occur independent of CpG methylation status (32), but at the same time has been shown to inhibit CpG methylation (38). Furthermore, DNA demethylation has previously been shown to occur when there are few methylated $\mathrm{CpG}$ sites within a CpG island, but not when all of the CpG sites are methylated (39), which is exactly the situation that we find for the FXN promoter region. However, the GAA 
repeat expansion-induced decreases in DNA methylation at the $F X N$ promoter are not consistent throughout all human and mouse brain and heart tissues, suggesting the involvement of other factors. Such factors may include differential susceptibility of the brain and heart tissues to DNA damage and/or GAA repeat instability. Indeed, FRDA is a disorder that is known to involve both oxidative DNA damage (40) and somatic instability of GAA repeats (3-5). Therefore, cells that are initially methylated at the $F X N$ promoter region may lose this methylation as part of the GAA repeat instability process, wherein demethylation subsequent to DNA damage repair (37) may be selected for due to beneficial effect of $F X N$ expression and hence cell viability. The GAA repeat expansion-induced decreases in DNA methylation that we have observed in the downstream GAA region of human tissues, but not transgenic mouse tissues, are more likely due to differently sized GAA repeats within the Alu sequence, as we have previously discussed. However, potential DNA demethylation in this downstream GAA region could also indirectly lead to an increase in $F X N$ transcription due to the removal of inhibitory effects on RNA polymerase II elongation.

Our investigations of histone modifications within FXN gene in both FRDA human and transgenic mouse brain tissues have now confirmed the changes previously reported for $\mathrm{H} 3$ and $\mathrm{H} 4$ deacetylation in the FXN promoter, upstream GAA and downstream GAA regions and H3K9 methylation in the upstream GAA region $(23,24)$. Furthermore, we have extended the H3K9 methylation analysis to include the $F X N$ promoter and downstream GAA regions that to our knowledge have not previously been reported for any FRDA tissue. Our findings from both 
human and transgenic mouse tissues indicate significant H3K9 deacetylation, which becomes more severe upon progression from the FXN promoter, through the upstream GAA region to the downstream GAA region. This correlates well with the results for both di- and tri-methylation of $\mathrm{H} 3 \mathrm{~K} 9$, which show a generally similar gradient of progressive increase from the FXN promoter, through the upstream GAA region, to the downstream GAA region. The only exception is the very high level of di-methylated H3K9 in FRDA brain tissue, which is higher than that in the downstream GAA region. All of the H3K9 changes correlate well with the DNA methylation changes in both human and transgenic mouse brain tissues. Thus, the patterns of progressively increasing H3K9 deacetylation and increasing $\mathrm{H} 3 \mathrm{~K} 9$ di- and tri-methylation in transgenic mouse brain correspond exactly to the pattern of increasing DNA methylation. Similarly, the patterns of progressively increasing $\mathrm{H} 3 \mathrm{~K} 9$ deacetylation and increasing $\mathrm{H} 3 \mathrm{~K} 9$ tri-methylation in human FRDA brain with a peak of $\mathrm{H} 3 \mathrm{~K} 9$ di-methylation in the upstream GAA region equate very well to the corresponding DNA methylation profiles. Therefore, our combined data thus far indicate major roles for DNA methylation, histone $\mathrm{H} 3 \mathrm{~K} 9$ deacetylation and histone $\mathrm{H} 3 \mathrm{~K} 9$ methylation in the inhibition of FXN transcription in brain and heart tissues, with a less prominent role for deacetylation of other histone residues. The more severe epigenetic changes within the $F X N$ intron 1 region compared with the promoter region support a hypothesis of transcription inhibition due to interference with elongation rather than initiation. Further work will be required to determine the exact relationships between DNA methylation, histone acetylation and methylation, heterochromatin 
formation and transcription inhibition. However, our results are consistent with the generally described pathway for gene inactivation wherein initial histone H3K9 deacetylation leads to H3K9 methylation, recruitment of HP1, histone deacetylases, DNA methyltransferases and eventual long-term shut down of transcription by DNA methylation (41). However, this situation is not likely to be universal for all trinucleotide repeat disorders, as highlighted by research on the FMR1 gene which has shown both histone deacetylation and H3K9 methylation in the absence of DNA methylation without interfering in active gene transcription (42).

For now, the exact mechanism by which the GAA repeat mutation inhibits frataxin expression remains elusive. However, accumulating evidence, including the findings of this report, now highlights the importance of epigenetic changes that lead to heterochromatin formation. The epigenetic changes that we and others have now identified in FRDA do not in any way negate the importance of any abnormal DNA or DNA/RNA hybrid structures in the inhibition of frataxin expression, but rather suggest the involvement of several combined mechanisms. Indeed the existence of abnormal DNA structures may help to explain why the GAA repeat mutation induces epigenetic changes in the first place. Thus, there are reports that non-B DNA structures such as hairpins may induce DNA methylation $(43,44)$, and GAA repeats have been shown to form hairpins (45). Alternatively, small double-stranded RNA (dsRNA) has also been shown to induce transcriptional gene silencing through a mechanism that involves DNA methylation $(46,47)$. However, dsRNA has failed to induce DNA 
methylation in a study of mouse oocytes (48) and dsRNA targeted to the $H D$ gene does not induce DNA methylation at the target huntingtin genomic locus in human cells (49). Thus, further studies are still required to identify any possible involvement of non-B DNA structures (such as GAA hairpins or triplex structures), DNA/RNA hybrids or dsRNA in the establishment of epigenetic changes and heterochromatin formation in FRDA.

In light of the epigenetic changes that we and others have identified in FRDA tissues and cells, several novel epigenetic-based therapeutic approaches can now be considered for FRDA. Firstly, histone deacetylase (HDAC) inhibitors can be used, and indeed these have already shown considerable promise by decreasing acetylation of histones and thereby increasing $F X N$ transcription in FRDA cells (24). Secondly, pharmacological approaches could be taken to decrease H3K9 methylation, as have recently been described for the combined use of mithramycin and cystamine in Huntington disease mice (50). Thirdly, therapies to decrease DNA methylation should now be considered for FRDA, as have previously been tried for other trinucleotide repeat disorders. In particular, 5-azadeoxycytidine (5-azadC) has been shown to remove DNA methylation of the CCG repeat expansion, increase $\mathrm{H} 3$ and $\mathrm{H} 4$ acetylation, decrease $\mathrm{H} 3 \mathrm{~K} 9$ methylation, increase $\mathrm{H} 3 \mathrm{~K} 4$ methylation and reactivate the $F M R 1$ gene $(30,51)$. Combined HDAC inhibitor and 5-azadC treatment has also been shown to synergistically increase FMR1 gene activity (52). Finally, short dsRNA molecules complementary to promoter sequences have recently been shown to induce gene activation $(53,54)$, and such approaches may also prove effective in 
increasing $F X N$ transcription. Our identification of a transgenic FRDA mouse model that shows comparable epigenetic changes to those seen in FRDA patients will now provide a valuable resource in the study of all such epigeneticbased FRDA therapies.

\section{MATERIALS AND METHODS}

Tissues. Human brain and heart tissue samples were obtained from autopsies of an FRDA patient (750/650 GAA repeats) and a non-FRDA individual, in accordance with UK Human Tissue Authority ethical guidelines. Mouse brain and heart tissues were dissected from our previously reported FXNYAC transgenic mouse models: Y47 (2 copies of 9 GAA repeats); YG8 (2 copies of 90 and 190 GAA repeats), and YG22 (1 copy of 190 GAA repeats) $(25,27)$.

mRNA expression analysis. Total RNA was isolated from frozen tissues by homogenization with Trizol (Invitrogen) and cDNA was then prepared by using AMV Reverse transcriptase (Invitrogen) with oligo-dT primers. Levels of human or mouse transgenic $F X N$ mRNA expression were assessed by quantitative RTPCR using an ABI7400 sequencer and SYBR ${ }^{\circledR}$ Green (Applied Biosystems) with the following primers: FxnRTF 5'-CAGAGGAAACGCTGGACTCT-3' and FxnRTR 5'-AGCCAGATTTGCTTGTTTGGC-3' (24). Human GAPDH or mouse Gapdh RT-PCR primers were used as control standards: human: GapdhhF 5'-GAAGGTGAAGGTCGGAGT-3' and GapdhhR 5'-GAAGATGGTGATGGGATTTC-3' 
mouse GapdhmF 5'-ACCCAGAAGACTGTGGATGG-3' and GapdhmR 5'GGATGCAGGGATGATGTTCT-3’

Bisulfite sequencing. Genomic DNA was isolated from frozen tissue by standard phenol/chloroform extraction and ethanol precipitation. 2 ug of genomic DNA was digested with EcoRI prior to bisulfite treatment using the CpGenome kit (Calbiochem). Nested PCR was carried out on bisulfite-treated DNA to amplify three regions of the $F X N$ gene using the following primers: Pro $1^{\text {st }}$ primer pair: SL1F1 5'-TAGTTTTTAAGTTTTTTTTTGTTTAG-3' and SL1R1 5'CAAAACAAAATATCCCCTTTTC-3'; Pro $2^{\text {nd }}$ primer pair: SL1F2 5'GTTTTTTTATAGAAGAGTGTTTG-3' and SL1R2 5'CAAAAACCAATATAAATACAACC-3'; Up 1 $^{\text {st }}$ primer pair: F1G 5'GAGGGATTTGTTTGGGTAAAG-3' and R1G 5'ATACTAAATTTCACCATATTAACC-3'; Up $2^{\text {nd }}$ primer pair: F2G 5'GATTTGTTTGGGTAAAGGTTAG-3' and R2G 5'CTCCCAAAATACTAAAATTATAAAC-3'; Down $1^{\text {st }}$ primer pair: NH1F 5'AAGAAGAAGAAGAAAATAAAGAAAAG-3' and SLGR2 5'TCCTAAAAAAAATCTAAAAACCATC-3'; Down $2^{\text {nd }}$ primer pair: NH2F 5'AGAAGAAGAAAATAAAGAAAAAGTTAG-3' and SLGR1 5'AAAACCATCATAACCACACTTAC-3'. PCR products were then resolved on agarose gels, purified with Geneclean (BIO101) and cloned into pCR4.0 (Invitrogen) prior to DNA sequencing. A minimum of 8 clones were sequenced for each tissue sample. 
ChIP analysis. Histone modifications at the three $F X N$ gene regions were detected by ChIP analysis of FRDA human and mouse tissues. This procedure involved initial cross-linking of DNA and protein by formaldehyde treatment of homogenised frozen tissue samples. DNA was then sheared by sonication, followed by immunoprecipitation with commercially available anti-histone and anti-acetylated histone $\mathrm{H} 3$ and $\mathrm{H} 4$ antibodies: $\mathrm{H} 3 \mathrm{~K} 9 \mathrm{ac}, \mathrm{H} 3 \mathrm{~K} 14 \mathrm{ac}, \mathrm{H} 4 \mathrm{~K} 5 \mathrm{ac}$, H4K8ac, H4K12ac, H4K16ac and H3K9me2 (Upstate), and H3K9me3 (Diagenode). For each experiment, normal rabbit serum (SIGMA) was used as a minus antibody immunoprecipitation control. After reversal of cross-linking, quantitative RT-PCR amplification of the resultant co-immunoprecipitated DNA was carried out with SYBR ${ }^{\circledR}$ Green in an ABI7400 sequencer (Applied Biosystems) using three sets of $F X N$ primers (Pro, Up and Down) and human GAPDH control for the human samples as previously described (24). For the analysis of transgenic mouse samples, the same three sets of $F X N$ primers were used together with the following mouse Gapdh control primers: GapdhMF, 5'TGACAAGAGGGCGAGCG-3' and GapdhMR, 5'-GGAAGCCGAAGTCAGGAAC-

3'. Each tissue sample was subjected to two independent ChIP procedures, followed by triplicate quantitative PCR analysis.

\section{ACKNOWLEDGEMENTS}

This research has been supported by the Friedreich's Ataxia Research Alliance (FARA), the National Ataxia Foundation (NAF), Ataxia UK, GoFAR and the King Faisal Specialist Hospital and Research Center, KSA. 


\section{CONFLICT OF INTEREST}

There is none declared.

\section{REFERENCES}

1. Campuzano, V., Montermini, L., Molto, M.D., Pianese, L., Cossee, M., Cavalcanti, F., Monros, E., Rodius, F., Duclos, F., Monticelli, A. et al. (1996) Friedreich's ataxia: autosomal recessive disease caused by an intronic GAA triplet repeat expansion. Science, 271, 1423-1427.

2. Pandolfo, M. (2002) The molecular basis of Friedreich ataxia. Adv. Exp. Med. Biol., 516, 99-118.

3. Clark, R.M., De Biase, I., Malykhina, A.P., Al-Mahdawi, S., Pook, M. and Bidichandani, S.I. (2006) The GAA triplet-repeat is unstable in the context of the human FXN locus and displays age-dependent expansions in cerebellum and DRG in a transgenic mouse model. Hum. Genet., 120, 633-640.

4. De Biase, I., Rasmussen, A., Endres, D., Al-Mahdawi, S., Monticelli, A., Cocozza, S., Pook, M. and Bidichandani, S.I. (2007) Progressive GAA expansions in dorsal root ganglia of Friedreich's ataxia patients. Ann. Neurol., 61, 55-60.

5. De Biase, I., Rasmussen, A., Monticelli, A., Al-Mahdawi, S., Pook, M., Cocozza, S. and Bidichandani, S.I. (2007) Somatic instability of the 
expanded GAA triplet-repeat sequence in Friedreich ataxia progresses throughout life. Genomics, 90, 1-5.

6. Campuzano, V., Montermini, L., Lutz, Y., Cova, L., Hindelang, C., Jiralerspong, S., Trottier, Y., Kish, S.J., Faucheux, B., Trouillas, P. et al. (1997) Frataxin is reduced in Friedreich ataxia patients and is associated with mitochondrial membranes. Hum. Mol. Genet., 6, 1771-1780.

7. Bulteau, A.L., O'Neill, H.A., Kennedy, M.C., Ikeda-Saito, M., Isaya, G. and Szweda, L.I. (2004) Frataxin acts as an iron chaperone protein to modulate mitochondrial aconitase activity. Science, 305, 242-245.

8. Gerber, J., Muhlenhoff, U. and Lill, R. (2003) An interaction between frataxin and Isu1/Nfs1 that is crucial for Fe/S cluster synthesis on Isu1. EMBO Rep., 4, 906-911.

9. Yoon, T. and Cowan, J.A. (2004) Frataxin-mediated iron delivery to ferrochelatase in the final step of heme biosynthesis. J. Biol. Chem., 279, 25943-25946.

10. Koeppen, A.H., Michael, S.C., Knutson, M.D., Haile, D.J., Qian, J., Levi, S., Santambrogio, P., Garrick, M.D. and Lamarche, J.B. (2007) The dentate nucleus in Friedreich's ataxia: the role of iron-responsive proteins. Acta. Neuropathol., 114, 163-173.

11. Hart, P.E., Lodi, R., Rajagopalan, B., Bradley, J.L., Crilley, J.G., Turner, C., Blamire, A.M., Manners, D., Styles, P., Schapira, A.H. et al. (2005) Antioxidant treatment of patients with Friedreich ataxia: four-year followup. Arch. Neurol., 62, 621-626. 
12. Mariotti, C., Solari, A., Torta, D., Marano, L., Fiorentini, C. and Di Donato, S. (2003) Idebenone treatment in Friedreich patients: one-year-long randomized placebo-controlled trial. Neurology, 60, 1676-1679.

13. Richardson, D.R., Mouralian, C., Ponka, P. and Becker, E. (2001) Development of potential iron chelators for the treatment of Friedreich's ataxia: ligands that mobilize mitochondrial iron. Biochim. Biophys. Acta., $1536,133-40$.

14. Rustin, P., Rotig, A., Munnich, A. and Sidi, D. (2002) Heart hypertrophy and function are improved by idebenone in Friedreich's ataxia. Free Radic. Res., 36, 467-469.

15. Schols, L., Vorgerd, M., Schillings, M., Skipka, G. and Zange, J. (2001) Idebenone in patients with Friedreich ataxia. Neurosci. Lett., 306, 169172.

16. Seznec, H., Simon, D., Monassier, L., Criqui-Filipe, P., Gansmuller, A., Rustin, P., Koenig, M. and Puccio, H. (2004) Idebenone delays the onset of cardiac functional alteration without correction of Fe-S enzymes deficit in a mouse model for Friedreich ataxia. Hum. Mol. Genet., 13, 1017-1024.

17. Bidichandani, S.I., Ashizawa, T. and Patel, P.I. (1998) The GAA tripletrepeat expansion in Friedreich ataxia interferes with transcription and may be associated with an unusual DNA structure. Am. J. Hum. Genet., 62, $111-121$

18. Grabczyk, E., Mancuso, M. and Sammarco, M.C. (2007) A persistent RNA.DNA hybrid formed by transcription of the Friedreich ataxia triplet 
repeat in live bacteria, and by T7 RNAP in vitro. Nucleic Acids Res., 35, $5351-5359$.

19. Grabczyk, E. and Usdin, K. (2000) The GAA*TTC triplet repeat expanded in Friedreich's ataxia impedes transcription elongation by T7 RNA polymerase in a length and supercoil dependent manner. Nucleic Acids Res., 28, 2815-2822.

20. Sakamoto, N., Ohshima, K., Montermini, L., Pandolfo, M. and Wells, R.D. (2001) Sticky DNA, a self-associated complex formed at long GAA*TTC repeats in intron 1 of the frataxin gene, inhibits transcription. J. Biol. Chem., 276, 27171-27177.

21. Saveliev, A., Everett, C., Sharpe, T., Webster, Z. and Festenstein, R. (2003) DNA triplet repeats mediate heterochromatin-protein-1-sensitive variegated gene silencing. Nature, 422, 909-913.

22. Egger, G., Liang, G., Aparicio, A. and Jones, P.A. (2004) Epigenetics in human disease and prospects for epigenetic therapy. Nature, 429, 457463.

23. Greene, E., Mahishi, L., Entezam, A., Kumari, D. and Usdin, K. (2007) Repeat-induced epigenetic changes in intron 1 of the frataxin gene and its consequences in Friedreich ataxia. Nucleic Acids Res., 35, 3383-3390.

24. Herman, D., Jenssen, K., Burnett, R., Soragni, E., Perlman, S.L. and Gottesfeld, J.M. (2006) Histone deacetylase inhibitors reverse gene silencing in Friedreich's ataxia. Nat. Chem. Biol., 2, 551-558. 
25. Al-Mahdawi, S., Pinto, R.M., Ruddle, P., Carroll, C., Webster, Z. and Pook, M. (2004) GAA repeat instability in Friedreich ataxia YAC transgenic mice. Genomics, 84, 301-310.

26. Al-Mahdawi, S., Pinto, R.M., Varshney, D., Lawrence, L., Lowrie, M.B., Hughes, S., Webster, Z., Blake, J., Cooper, J.M., King, R. et al. (2006) GAA repeat expansion mutation mouse models of Friedreich ataxia exhibit oxidative stress leading to progressive neuronal and cardiac pathology. Genomics, 88, 580-590.

27. Pook, M.A., Al-Mahdawi, S., Carroll, C.J., Cossee, M., Puccio, H., Lawrence, L., Clark, P., Lowrie, M.B., Bradley, J.L., Cooper, J.M. et al. (2001) Rescue of the Friedreich's ataxia knockout mouse by human YAC transgenesis. Neurogenetics, 3, 185-193.

28. Greene, E., Handa, V., Kumari, D. and Usdin, K. (2003) Transcription defects induced by repeat expansion: fragile $X$ syndrome, FRAXE mental retardation, progressive myoclonus epilepsy type 1, and Friedreich ataxia. Cytogenet. Genome Res., 100, 65-76.

29. Steinbach, P., Glaser, D., Vogel, W., Wolf, M. and Schwemmle, S. (1998) The DMPK gene of severely affected myotonic dystrophy patients is hypermethylated proximal to the largely expanded CTG repeat. Am. J. Hum. Genet., 62, 278-285.

30. Tabolacci, E., Pietrobono, R., Moscato, U., Oostra, B.A., Chiurazzi, P. and Neri, G. (2005) Differential epigenetic modifications in the FMR1 gene of 
the fragile $\mathrm{X}$ syndrome after reactivating pharmacological treatments. Eur. J. Hum. Genet., 13, 641-648.

31. Graff, J.R., Herman, J.G., Myohanen, S., Baylin, S.B. and Vertino, P.M. (1997) Mapping patterns of CpG island methylation in normal and neoplastic cells implicates both upstream and downstream regions in de novo methylation. J. Biol. Chem., 272, 22322-22329.

32. Clark, S.J., Harrison, J. and Molloy, P.L. (1997) Sp1 binding is inhibited by (m) $\mathrm{Cp}(\mathrm{m}) \mathrm{CpG}$ methylation. Gene, 195, 67-71.

33. Greene, E., Entezam, A., Kumari, D. and Usdin, K. (2005) Ancient repeated DNA elements and the regulation of the human frataxin promoter. Genomics, 85, 221-230.

34. Matsuo, K., Silke, J., Georgiev, O., Marti, P., Giovannini, N. and Rungger, D. (1998) An embryonic demethylation mechanism involving binding of transcription factors to replicating DNA. Embo J., 17, 1446-1453.

35. Weiss, A., Keshet, I., Razin, A. and Cedar, H. (1996) DNA demethylation in vitro: involvement of RNA. Cell, 86, 709-718.

36. Turk, P.W., Laayoun, A., Smith, S.S. and Weitzman, S.A. (1995) DNA adduct 8-hydroxyl-2'-deoxyguanosine (8-hydroxyguanine) affects function of human DNA methyltransferase. Carcinogenesis, 16, 1253-1255.

37. Cuozzo, C., Porcellini, A., Angrisano, T., Morano, A., Lee, B., Pardo, A.D., Messina, S., luliano, R., Fusco, A., Santillo, M.R. et al. (2007) DNA Damage, Homology-Directed Repair, and DNA Methylation. PLoS Genet., 3, e110. First published on May 22, 2007, 10.1371/pgen.0030110. 
38. Macleod, D., Charlton, J., Mullins, J. and Bird, A.P. (1994) Sp1 sites in the mouse aprt gene promoter are required to prevent methylation of the $\mathrm{CpG}$ island. Genes. Dev., 8, 2282-2292.

39. Choi, Y.C. and Chae, C.B. (1993) Demethylation of somatic and testisspecific histone $\mathrm{H} 2 \mathrm{~A}$ and $\mathrm{H} 2 \mathrm{~B}$ genes in F9 embryonal carcinoma cells. Mol. Cell. Biol., 13, 5538-5548.

40. Schulz, J.B., Dehmer, T., Schols, L., Mende, H., Hardt, C., Vorgerd, M., Burk, K., Matson, W., Dichgans, J., Beal, M.F. et al. (2000) Oxidative stress in patients with Friedreich ataxia. Neurology, 55, 1719-1721.

41. D'Alessio, A.C. and Szyf, M. (2006) Epigenetic tete-a-tete: the bilateral relationship between chromatin modifications and DNA methylation. Biochem. Cell Biol., 84, 463-476.

42. Pietrobono, R., Tabolacci, E., Zalfa, F., Zito, I., Terracciano, A., Moscato, U., Bagni, C., Oostra, B., Chiurazzi, P. and Neri, G. (2005) Molecular dissection of the events leading to inactivation of the FMR1 gene. Hum. Mol. Genet., 14, 267-277.

43. Chen, X., Mariappan, S.V., Catasti, P., Ratliff, R., Moyzis, R.K., Laayoun, A., Smith, S.S., Bradbury, E.M. and Gupta, G. (1995) Hairpins are formed by the single DNA strands of the fragile $X$ triplet repeats: structure and biological implications. Proc. Natl. Acad. Sci. USA, 92, 5199-5203.

44. Laayoun, A. and Smith, S.S. (1995) Methylation of slipped duplexes, snapbacks and cruciforms by human DNA(cytosine-5)methyltransferase. Nucleic Acids Res., 23, 1584-1589. 
45. Heidenfelder, B.L., Makhov, A.M. and Topal, M.D. (2003) Hairpin formation in Friedreich's ataxia triplet repeat expansion. J. Biol. Chem., 278, 2425-2431.

46. Kawasaki, H. and Taira, K. (2004) Induction of DNA methylation and gene silencing by short interfering RNAs in human cells. Nature, 431, 211-217.

47. Morris, K.V., Chan, S.W., Jacobsen, S.E. and Looney, D.J. (2004) Small interfering RNA-induced transcriptional gene silencing in human cells. Science, 305, 1289-1292.

48. Svoboda, P., Stein, P., Filipowicz, W. and Schultz, R.M. (2004) Lack of homologous sequence-specific DNA methylation in response to stable dsRNA expression in mouse oocytes. Nucleic Acids Res., 32, 3601-3606.

49. Park, C.W., Chen, Z., Kren, B.T. and Steer, C.J. (2004) Double-stranded siRNA targeted to the huntingtin gene does not induce DNA methylation. Biochem. Biophys. Res. Commun., 323, 275-280.

50. Ryu, H., Lee, J., Hagerty, S.W., Soh, B.Y., McAlpin, S.E., Cormier, K.A., Smith, K.M. and Ferrante, R.J. (2006) ESET/SETDB1 gene expression and histone $\mathrm{H} 3$ (K9) trimethylation in Huntington's disease. Proc. Natl. Acad. Sci. USA, 103, 19176-19181.

51. Pietrobono, R., Pomponi, M.G., Tabolacci, E., Oostra, B., Chiurazzi, P. and Neri, G. (2002) Quantitative analysis of DNA demethylation and transcriptional reactivation of the FMR1 gene in fragile $X$ cells treated with 5-azadeoxycytidine. Nucleic Acids Res., 30, 3278-3285. 
52. Chiurazzi, P., Pomponi, M.G., Pietrobono, R., Bakker, C.E., Neri, G. and Oostra, B.A. (1999) Synergistic effect of histone hyperacetylation and DNA demethylation in the reactivation of the FMR1 gene. Hum. Mol. Genet., 8, 2317-2323.

53. Janowski, B.A., Younger, S.T., Hardy, D.B., Ram, R., Huffman, K.E. and Corey, D.R. (2007) Activating gene expression in mammalian cells with promoter-targeted duplex RNAs. Nat. Chem. Biol., 3, 166-173.

54. Li, L.C., Okino, S.T., Zhao, H., Pookot, D., Place, R.F., Urakami, S., Enokida, H. and Dahiya, R. (2006) Small dsRNAs induce transcriptional activation in human cells. Proc. Natl. Acad. Sci. USA, 103, 17337-17342.

\section{LEGENDS TO FIGURES}

Figure 1. Quantitative RT-PCR analysis of FXN mRNA isolated from brain and heart autopsy samples of an FRDA patient (750 and 650 GAA repeats) and an unaffected individual. Data are normalised to the FXN mRNA level found in the unaffected individual. Two individual cDNA samples were analysed for each tissue and each reaction was carried out in triplicate. The means and SEMs of these values are shown.

Figure 2. Schematic representation of $2.2 \mathrm{~kb}$ at the $5^{\prime}$ end of the $F X N$ gene, indicating the promoter / exon 1 (Pro), upstream GAA (Up) and downstream GAA (Down) regions that were analysed by ChIP (black boxes) and bisulfite sequencing (hatched boxes). Numbers above indicate the position of $\mathrm{CpG}$ sites 
within the promoter and upstream GAA regions. The positions of the ATG translation start codon, exon 1 open reading frame and GAA repeat sequence within the Alu repeat sequence are shown. Numbers below indicate the chromosome 9 base pair numbering according to the 2006 build of the UCSC human DNA sequence database.

Figure 3. DNA methylation analysis of the $F X N$ promoter (A, B), upstream GAA (C, D) and downstream GAA (E, F) regions of human brain and heart tissues. In each case the percentage of methylated $\mathrm{CpG}$ sites is shown as determined from the analysis of 8 to 12 independent cloned DNA sequences. Only eleven CpG sites are represented for the promoter region (A, B), as sites 11-22 and 24-59 did not show any methylation in either FRDA or unaffected samples in brain or heart.

Figure 4. Analysis of histone modifications in human brain tissue. ChIP quantitative PCR results for the $F X N$ promoter/exon1 (Pro), upstream GAA (Up) and downstream GAA (Down) amplified regions are represented as the relative amount of immunoprecipitated DNA compared with input DNA, having taken negligible $-\mathrm{Ab}$ control values into account. $F X N$ values were normalised with human GAPDH and all values have been adjusted so that all of the Upstream GAA values from the unaffected individual are $100 \%$. In each case two individual ChIP samples were analysed in triplicate. The means and SEMs of these values are shown. 
Figure 5. Quantitative RT-PCR analysis of transgenic FXN mRNA isolated from Y47, YG8 and YG22 mouse brain and heart tissues. Data are normalised to the FXN mRNA level found in the non-GAA transgenic mouse. Two individual cDNA samples were analysed for each tissue and each reaction was carried out in triplicate. The means and SEMs of these values are shown.

Figure 6. DNA methylation analysis of the $F X N$ promoter $(A, B)$, upstream GAA (C, D) and downstream GAA (E, F) regions of YG22 (GAA) and Y47 (non-GAA) transgenic mouse brain and heart tissues. In each case the percentage of methylated $\mathrm{CpG}$ sites is shown as determined from the analysis of 8 to 25 independent cloned DNA sequences. Only eleven CpG sites are represented for the promoter region, as sites 11-22 and 24-59 did not show any methylation in either FRDA or unaffected samples in brain or heart.

Figure 7. Analysis of histone modifications in transgenic mouse brain tissue. ChIP quantitative PCR results for the transgenic FXN promoter/exon1 (Pro), upstream GAA (Up) and downstream GAA (Down) amplified regions are represented as the relative amount of immunoprecipitated DNA compared with input DNA, having taken negligible -Ab control values into account.. $F X N$ values were normalised with mouse GAPDH and all values have been adjusted so that all of the upstream GAA values from the non-GAA transgenic mouse (Y47) are $100 \%$. In each case two individual ChIP samples were analysed in triplicate. The means and SEMs of these values are shown. 
Fig. 1

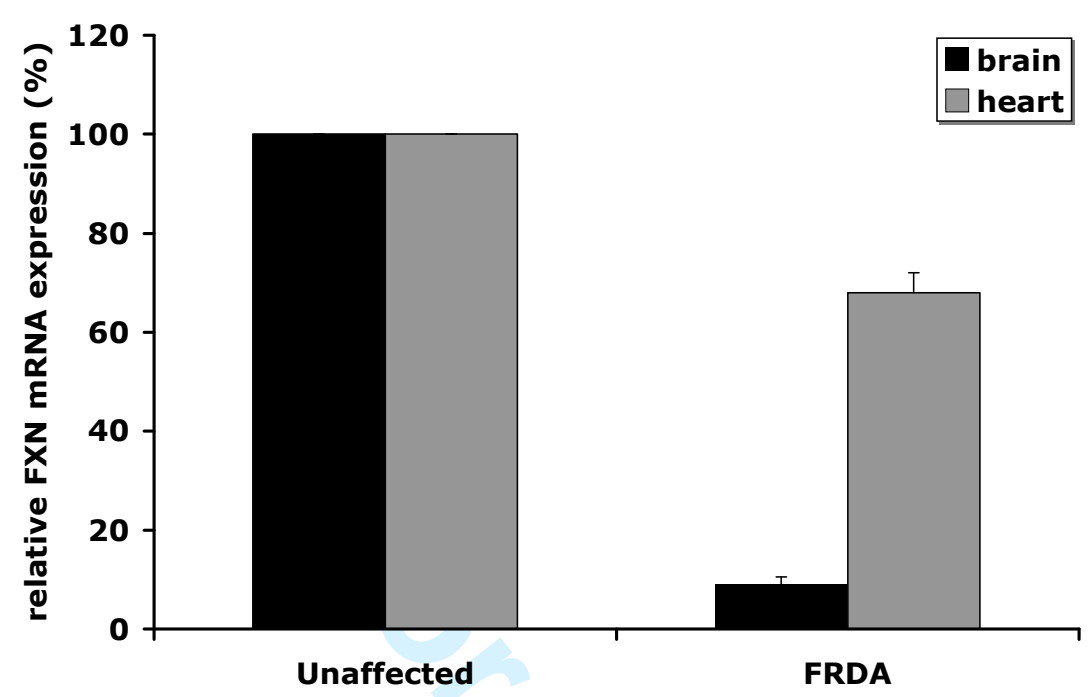

Fig.2

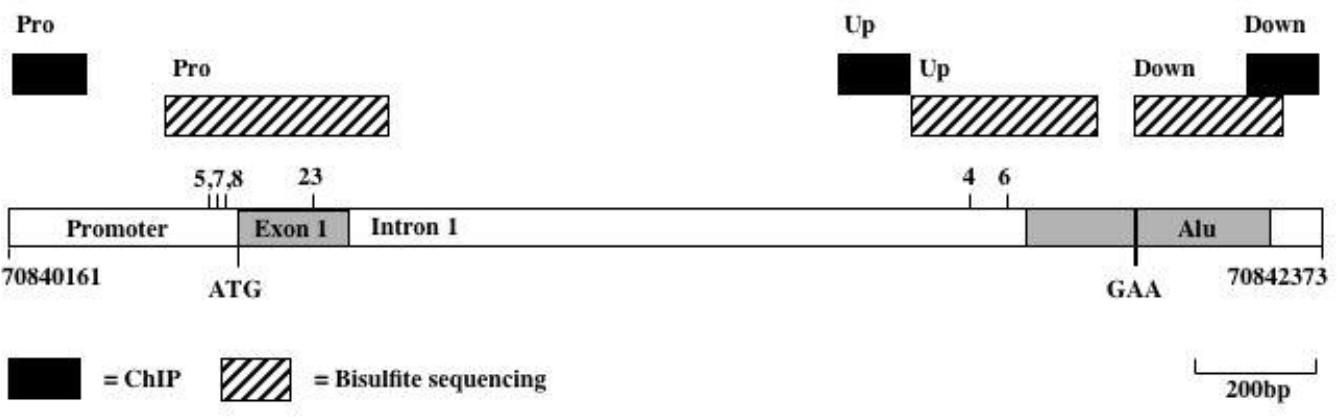


Fig. 3
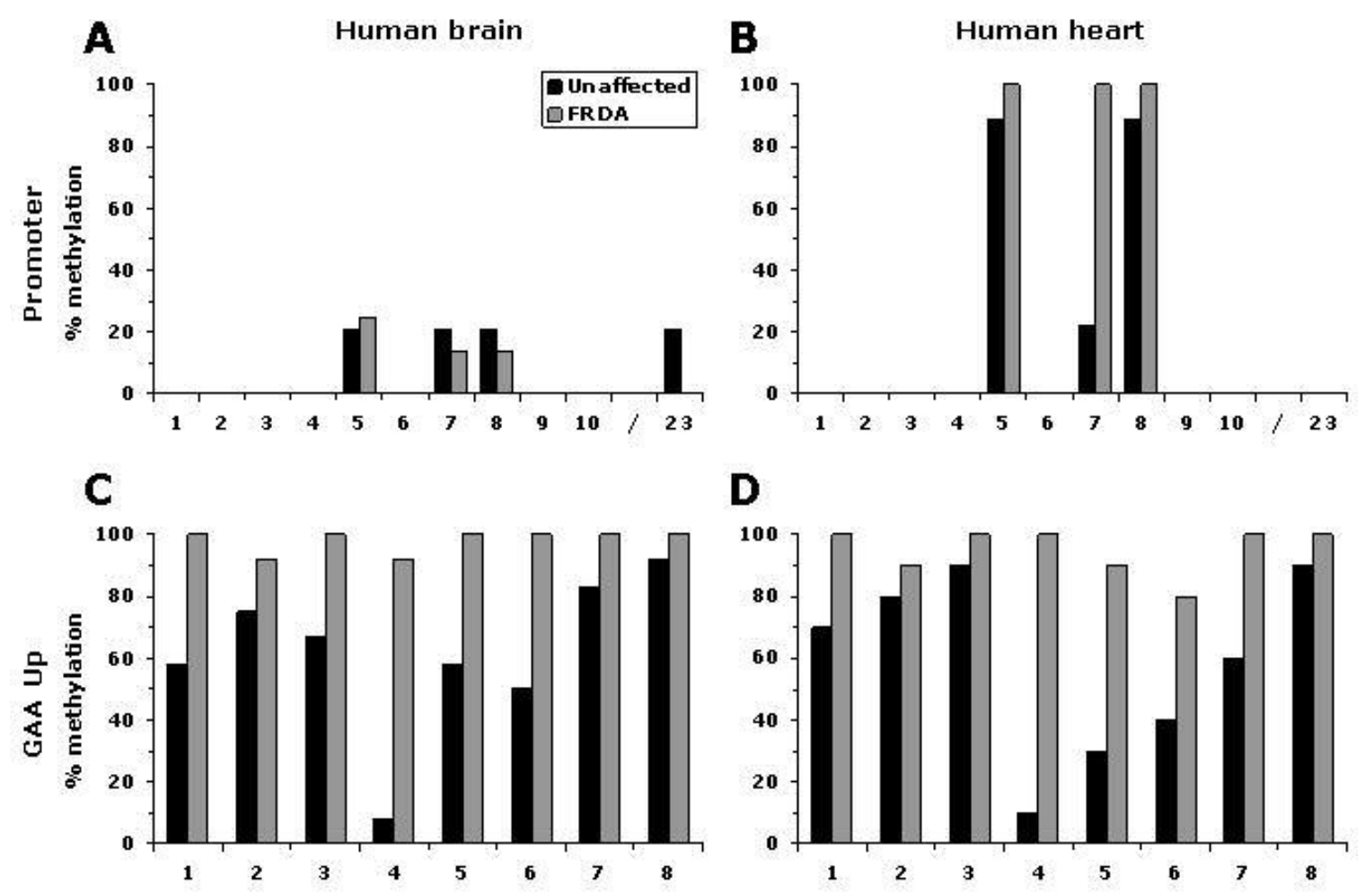

E

F
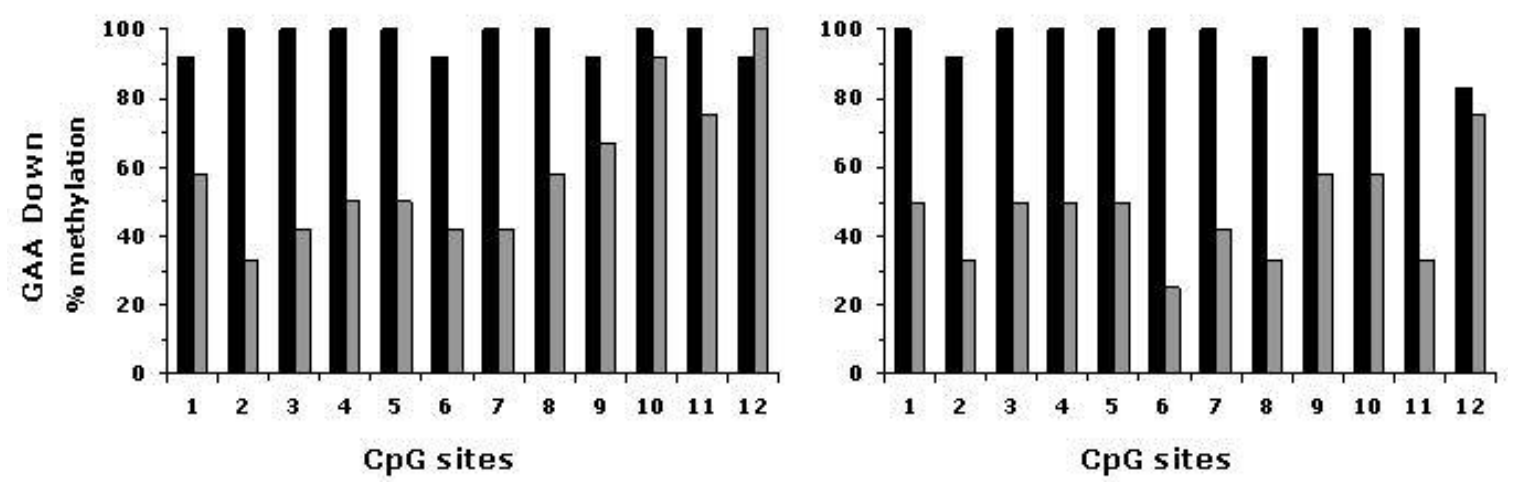
Fig. 4

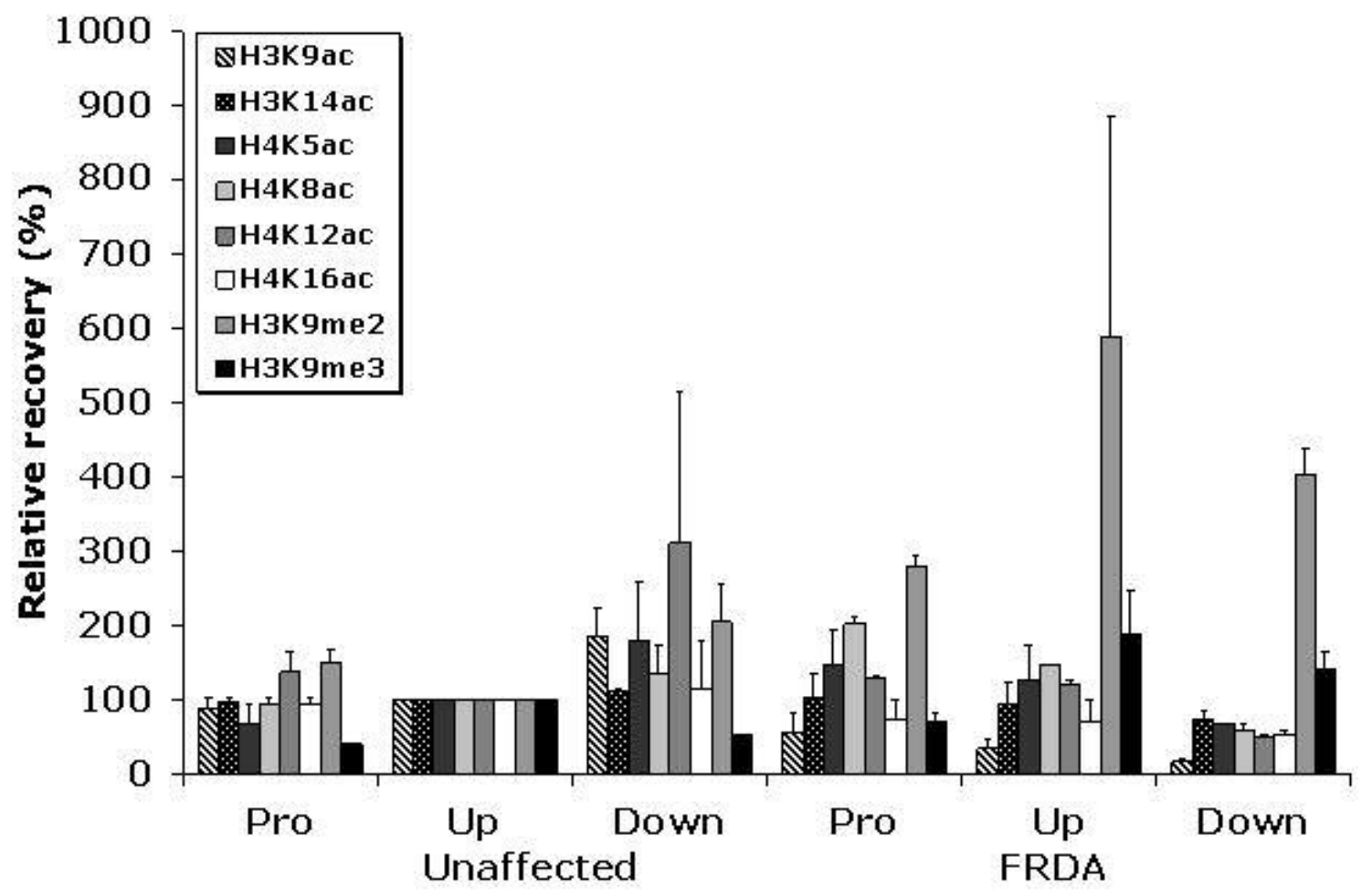

Fig. 5

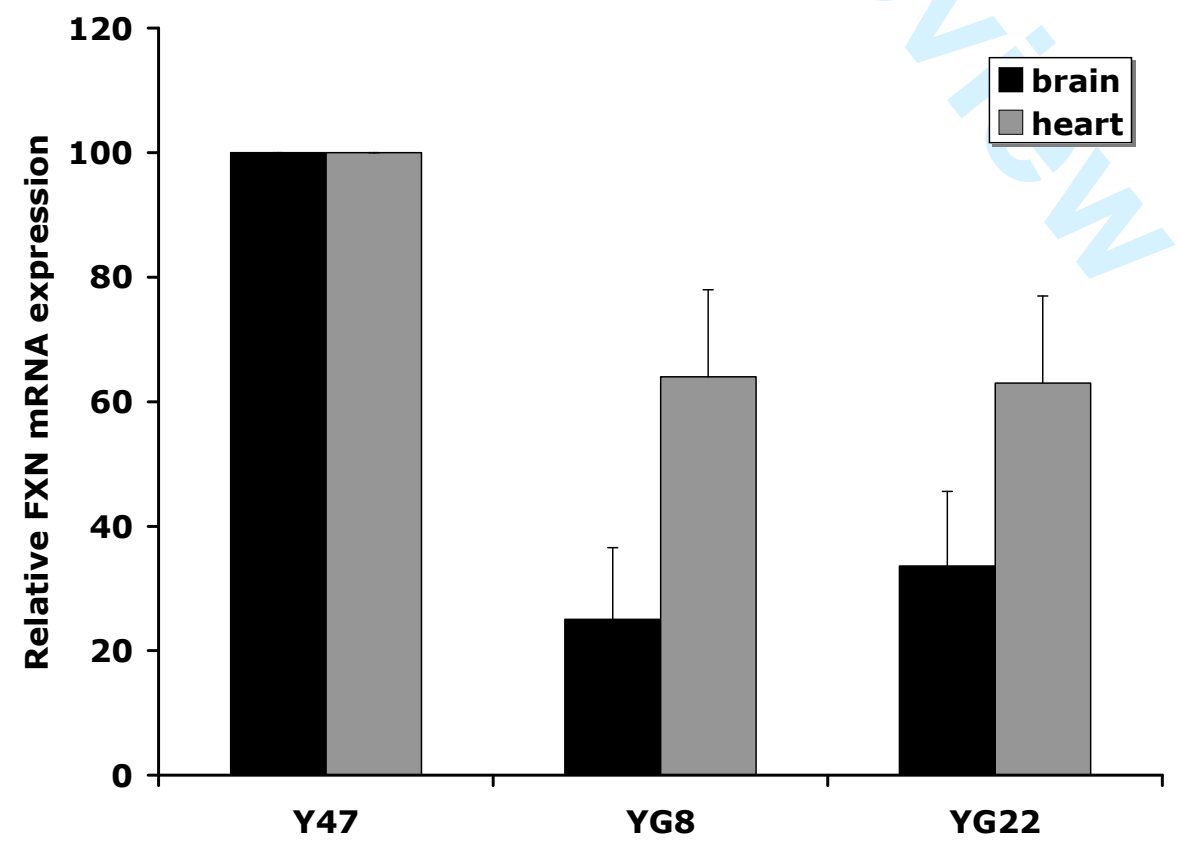


Fig. 6
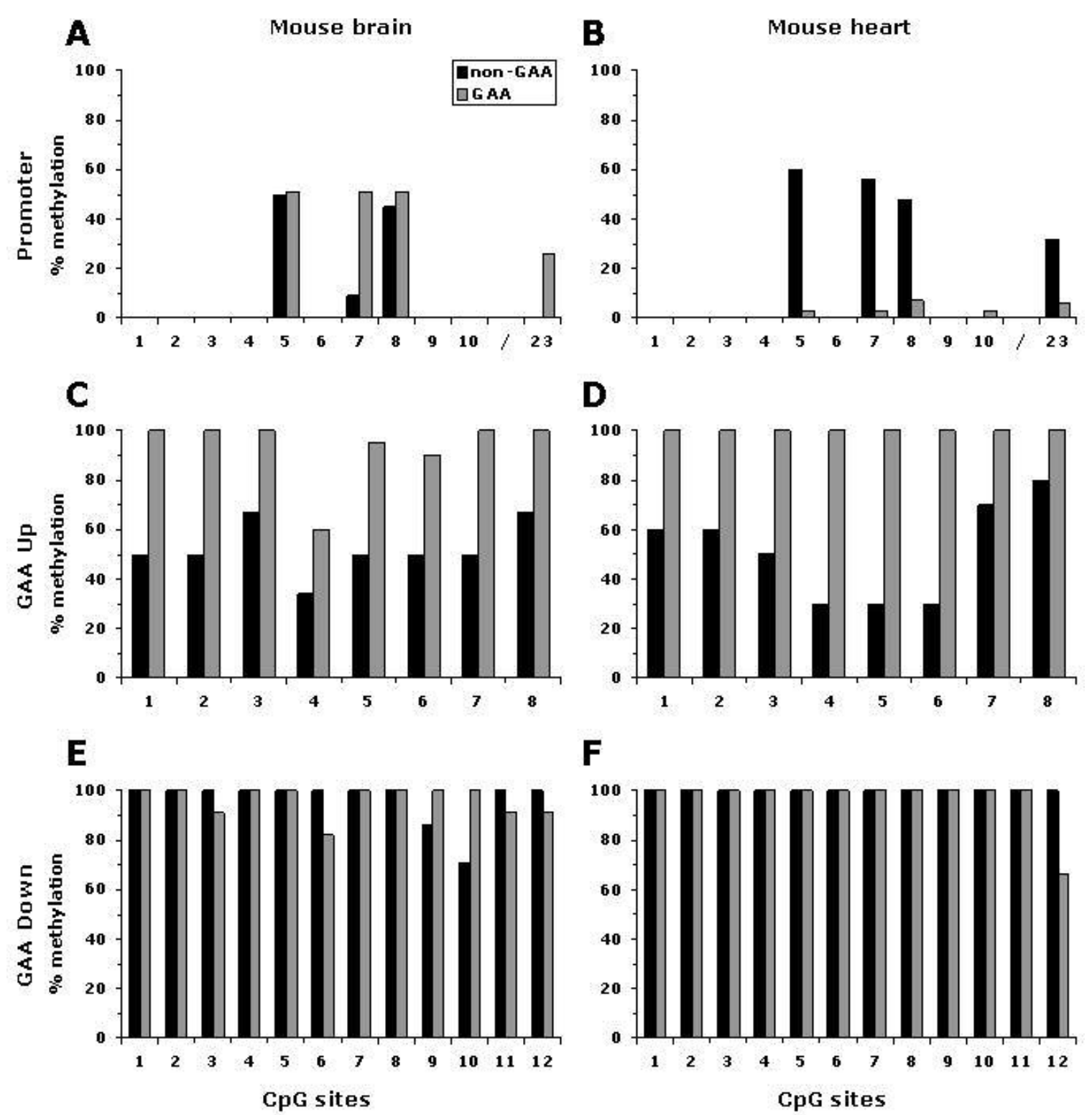

$F$

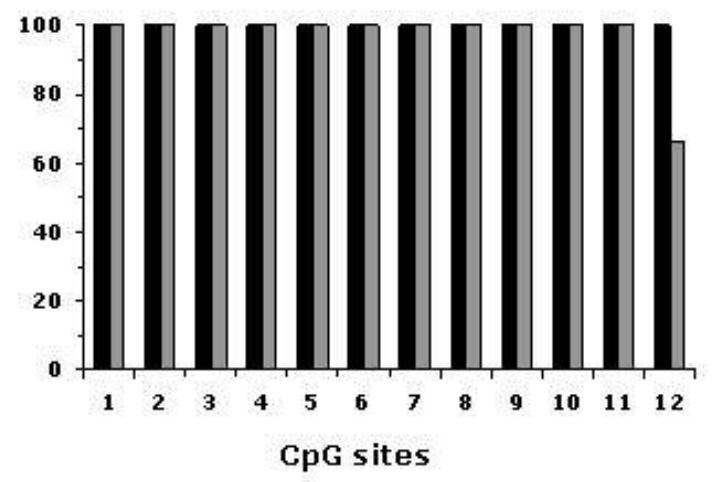


Fig.7

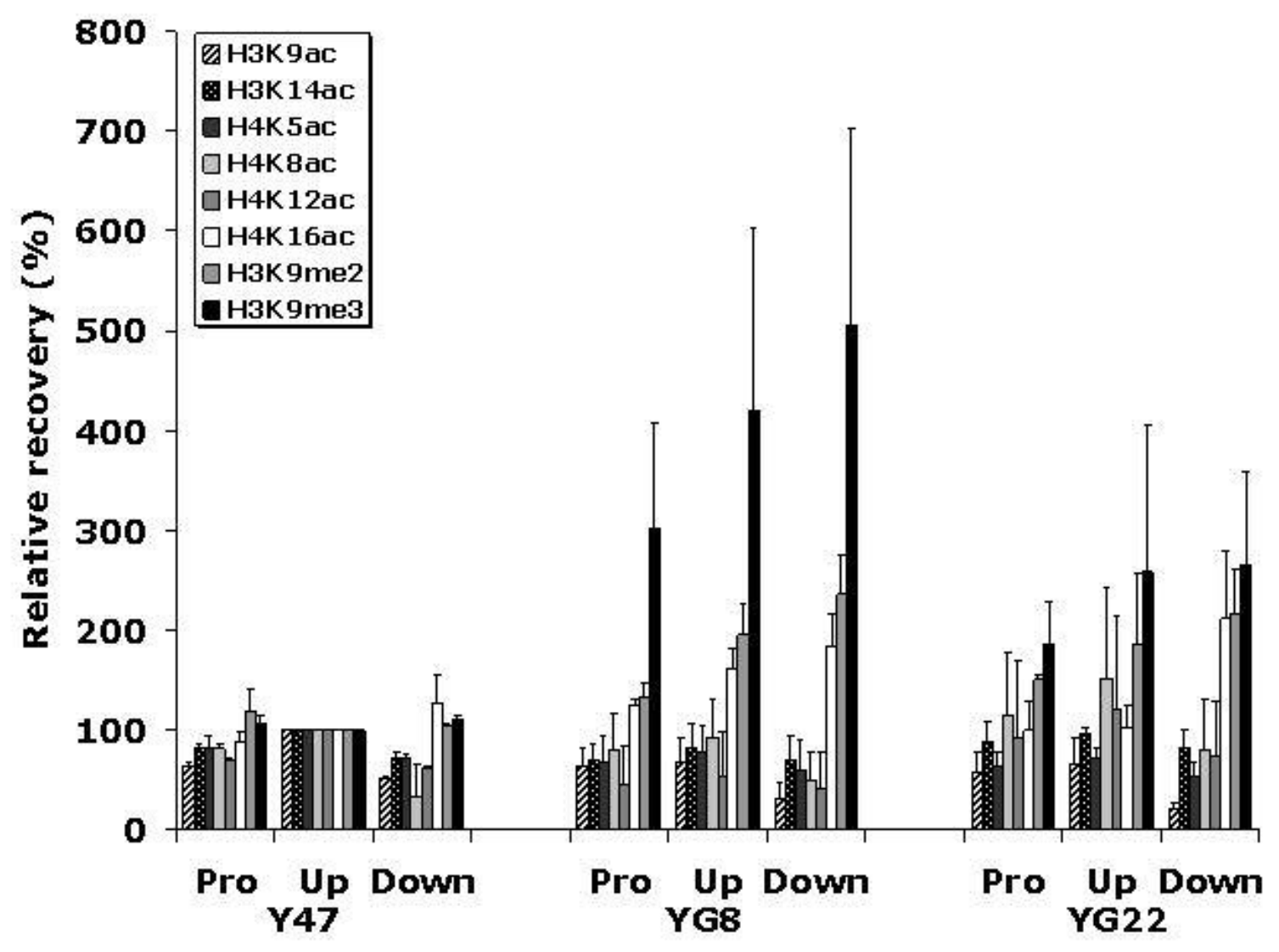




\section{ABBREVIATIONS}

5-azadC: 5-azadeoxycytidine; 8-OH-dG: 8-hydroxyl-2'-deoxyguanosine; Ab: antibody; ChIP: chromatin immunoprecipitation; DRG: dorsal root ganglia; FRDA: Friedreich ataxia; GAPDH: glyceraldehyde-3-phosphate dehydrogenase; H3K9: histone 3 lysine 9 residue; HDAC: histone deacetylase; HP1: heterochromatin protein 1; RT-PCR: reverse transcriptase polymerase chain reaction; SEM: standard error of the mean; Sp1: specificity protein 1. 